\title{
The Double Consciousness Created by Male Gaze Upon Young Women in Chinese Society
}

\author{
Zixuan Wang ${ }^{1, *}$ \\ ${ }^{1}$ The Experimental High School attached to Beijing Normal University \\ *Corresponding author. Email: pitcvo@163.com
}

\begin{abstract}
Male gaze, originally a common phenomenon in films, can also be applied to daily life. In modern Chinese society, male gaze is giving rise to a sense of double consciousness among young females, which indicates that their real self is constantly conflicting with and even being repressed by the other self that is dominated by the gaze from males.

Data from social media and online database demonstrate the impact of the double consciousness on young female individuals from the aspects of body anxiety, workplace inequality, and family roles. By analyzing the status quo of young females in China, this paper illustrates how male gaze is actually affecting them by undermining their recognition of their real self - their individuality and their identity-- in their subconscious.

Although analysis of specific film suggests efforts of females to avoid male gaze, it is still hard to raise the awareness of this double consciousness due to the history of Chinese feudal society that has long oppressed the nature of women.
\end{abstract}

Keywords: Male Gaze, Double Consciousness, Young Women in Chinese Society, Conflict of Identity

\section{INTRODUCTION}

When watching mainstream films, the female characters' glamorous dresses and the jokes related to sex often bring the female audience a slight discomfort. Most of this discomfort in films derives from the perspective from which the director portrays the character - the male gaze, a concept of male gaze first used in film criticism by Laura Mulvey, a feminist film theorist from Britain.

The purpose of this paper is to study the male gaze and its effects upon young Chinese women, so as to arouse the cognition of the society on the phenomena of male gaze, and to call on women to be aware of and get rid of its influences.

The significance of this study lies in examining male gaze, an original film theory, in a real life context, and analyzing how male gaze exerts considerable impact on women utilizing the double consciousness theory of Du Bois, as previous studies rarely compare male gaze in movies with that in real life, nor do they search for the connection between double consciousness theory and male gaze.

\section{LITERATURE REVIEW}

\subsection{Male Gaze}

The concept of male gaze was first used in film criticism by Laura Mulvey, a feminist film theorist from Britain, in her work "Visual Pleasure and Narrative Movies" written in 1973 [1]. It was initially a concept in film making which states that in movies, men often take the dominant place and act as the subject of gazing, while women usually become the object of gazing. This phenomena stems from the fact that in the 1970s, film making was a highly male-dominated industry where almost all directors, cinematographers, and producers were males. The protagonist is usually a man, and more importantly, the target audiences are also men. In other words, most movies are aimed at serving men' $s$ tastes. While several men are chatting, an attractive female makes a stunning debut and instantly attracts the eyes of all men around. Subsequently, the camera follows the males' gaze, gradually showing the female' $\mathrm{s}$ body from toe to head. This is a classic example of male gaze. Although an increasing number of modern movies are trying to feature female protagonists, the basic concept of male gaze still holds true today [2]. 
Art Critic John Berger pointed out that "Men look at women. Women watch themselves being looked at. This determines not only most relations between men and women but also the relation of women to themselves" [3], which perfectly illustrates how male gaze is exerted upon women and how women are being influenced.

\subsection{Double Consciousness}

The theory of double consciousness, proposed by William Edward Burghardt Du Bois, originally refers to the two distinct identities an African American experiences in a white-dominant society. Du Bois defines double consciousness in his work The Souls of Black Folks as follows:

It is a peculiar sensation, this double-consciousness, this sense of always looking at one' s self through the eyes of others, of measuring one' $\mathrm{s}$ soul by the tape of a world that looks on in amused contempt and pity. One ever feels his two-ness, - an American, a Negro; two souls, two thoughts, two unreconciled strivings; two warring ideals in one dark body, whose dogged strength alone keeps it from being torn asunder.

This theory describes the conflict a black individual faces in recognizing his or her identity in a society, and how the influence of a predominantly white society on black people result in struggle.

\section{HYPOTHESIS}

In modern context, the application of double consciousness can be extended beyond racial problems. I argue that the hegemony of the male gaze creates a type of gender-based double consciousness upon young women in Chinese society. In fact, a double consciousness is being demonstrated in various aspects, which can lead to significantly destructive impact on the life of the young female generation in China.

\section{METHODOLOGY}

\subsection{Gathering Data from Social Media}

Evidence on the impact of male gaze on women's body anxiety is collected from the "Weibo" and "Xiaohongshu", the largest social medium in China with over 400 million users, by searching, viewing posted content, and counting word frequencies. Since the function of "Weibo" and "Xiaohongshu" is to share daily life publicly, and most of the social medium users are teenagers as well as young adults, the data collected can reflect the real life and thoughts of today's young women in China and demonstrate the general trend of how young women are being affected by male gaze in the aspect of physical shape.

\subsection{Using Data from Online Database}

The analysis of the impact of male gaze on women in the workplace derives from "Investigation Report on the Status Quo of Chinese Women's Workplace in 2020", a survey conducted by Zhilian Recruitment. This is a relatively recent survey, with detailed data that includes many different perspectives on the current status of women in the workplace, such as the most common female occupations and the percentage of high level positions among females.

\subsection{Analyzing Specific Film}

As the male gaze was originally a film theory, in order to demonstrate the language of the camera in films, the paper includes brief analysis of a specific film to demonstrate the attempt of female directors to break through the tradition of male gaze.

\section{ANALYSIS}

\subsection{One Part of the Double Consciousness: The Internalized Male Gaze}

\subsubsection{Body Anxiety}

The most obvious manifestation is the fanatical pursuit of slim body shape in recent years. The society' $s$ aesthetic standards for women are often higher than those for men, rendering women under constant scrutinization. Chinese society' $\mathrm{s}$ excessive pursuit for slimness has led to such a deformed aesthetic view that losing weight has become the consensus of almost all young women. This overly demanding aesthetic standard can be explained as the society' s internalization of men' $\mathrm{s}$ expectations. A certain behavior can easily be defined as "wrong" for it violates "the assumptions of the likely viewer" [3]. To cater to the "ideal" beauty standard set by males as well as the whole society, women can readily feel disgust towards their own body, and even employ some extreme methods to achieve the "perfect" body shape: they tend to construct themselves considering the gaze from males.

In the past two years, some "body challenges" have become a prevailing trend, causing many young women to imitate. These body challenges are not challenges to physical strength or fitness, but are in fact displays of their attractive body parts to the public via social media. One of these challenges is the "A4 waist" challenge, where young ladies compare their waists to the width of a A4 paper in order to show that they have got slim waists. On a social networking site named "Weibo" , over 200 females under age 29 include A4 waist in their usernames to imply their 
dream of having an A4 waist. On another social application called "Xiaohongshu", there are over 10,000 posts sharing photos of their A4 waists as well as 60 kinds of related good being sold in the online market in the app; furthermore, there are tags such as "A4 waist crash course" where girls teach others how to gain an extremely slim waist curve. Some even use corsets (figure 1) to physically tighten their curve. There are even more posts sharing information on corsets, reaching the number of 50,000, with 3300 related goods on sale. As wearing such corsets has no benefits to the women' $\mathrm{s}$ body and may even result in irreversible damage to the waist, this behavior is not to promote themselves, but to meet male' $\mathrm{s}$ aesthetics standards. This trend is an illustration of how young women in China are devoting themselves to pander to male gaze and constructing themselves based on the expectations of males rather than their own personal values.

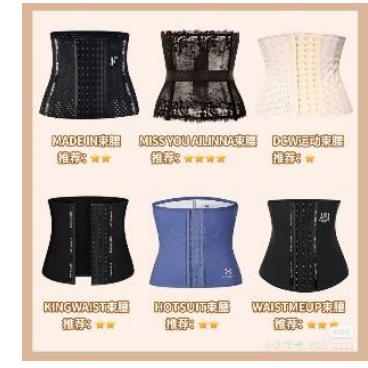

Figure 1 A Post Ranking Different Corsets

Table 1. Number of Posts and Goods on Sale related to A4 Waist Photos and Corsets on Xiaohongshu

\begin{tabular}{|c|c|c|}
\hline & A4 Waist Photos & Corsets \\
\hline $\begin{array}{c}\text { Number of } \\
\text { Posts }\end{array}$ & $10,000+$ & 60 \\
\hline $\begin{array}{c}\text { Number of } \\
\text { Goods on Sale }\end{array}$ & $50,000+$ & 3,300 \\
\hline
\end{tabular}

\subsubsection{Workplace Inequality}

In addition, women in China are profoundly affected by double consciousness in terms of career choice and workplace status. From the perspective of some males, women's persistence, strength, leadership skills and determination are generally weaker than those of men. Thus, women are considered unsuitable for certain positions or unable to assume the role of leader, which in turn makes women themselves think that certain traditional female occupations, such as nurses and teachers, are their only pathways, or that they are inherently inferior to the male leaders. This conception would limit their future development and inhibit their original potential.
According to the "Investigation Report on the Status Quo of Chinese Women's Workplace in 2020" conducted by Zhilian Recruitment [4], it is explicit that occupations also have gender attributes. The proportion of female workers in service industry is significantly higher than that of men, which include logistics management, administration, secretary, etc., while highly technical occupations are the world of men.

Moreover, women are still in the passive following role in leadership activities in the workplace. In different levels of manager positions, the proportion of women is generally lower than that of men, especially in the position of senior executives: $9 \%$ of men are in senior executive positions, while only $5 \%$ of women are on the same level. Similarly, $46.3 \%$ women are general staff (who usually acquire less salary and lower status in workplace), while only $31 \%$ men take the same basic position. Even if men and women achieve the ideal state of equal pay for equal work, the general absence of women in leadership roles still limits women' s voice.

\section{Distribution of Positions in Workplace}

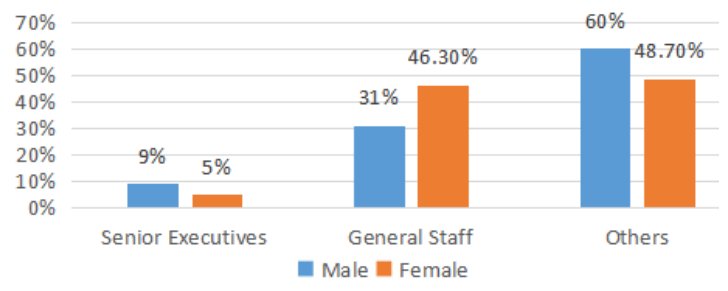

Figure 2 Distribution of Positions in Workplace among Different Genders

\subsubsection{Conventional Family Roles}

Besides the pressure in workplace, women also suffer from male gaze in their families, for China has been a patriarchal society for thousands of years. Even in the 21 st century, a number of traditional Chinese families follow lots of conventions inherited from the feudal society, some of which impose heavy shackles and burdens on women, destroying the construction of young women's own unique identity. In Chinese society, there are "the three obediences and the four virtues" for women to follow, which are traditional moral standards for women in feudal society. The three obediences are: obeying to father before marriage, obeying to husband after marriage, and obeying to son after the demise of husband. The four virtues include morality, proper speech, modest manner and diligent housework. Some families still hold this kind of conservative concept, suppressing women' $\mathrm{s}$ personalities and rendering them the product of male gaze. More commonly, parents and husbands would not evidently ask the wife to follow such rules, but the stereotype that a woman ought to carry out all the 
housework, raise the children, and serve all other family members still exist and has rooted deeply in the minds of not only men but also women themselves, who subconsciously recognizes such roles and automatically assumes these tasks.

\subsection{The Other Part of the Double Consciousness}

\subsubsection{The Real Self}

In the double consciousness created upon young Chinese women, there is the other part of the "double", that is, the one with which the maledominated self co-exists. However, under the influence of the male gaze, this true self is being repressed and neglected. This kind of ignorance is not only the ignorance from males, but also the ignorance among females of their original identity. A female individual may possess unique charm, unlimited potential, and outstanding personal ability, but these qualities constantly conflict with the male-dominated self and cannot be fully displayed. As long as male gaze exists and the division between the two parts of double consciousness exist, such inner struggle will continue to repress the nature of women, and women will not be able to establish a truly integrated and independent identity of their own.

\subsubsection{Women's Awakening}
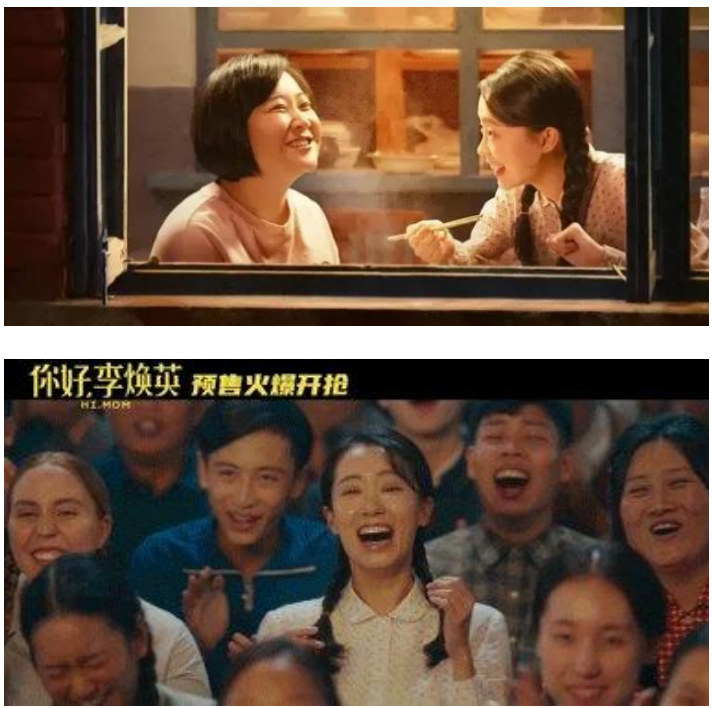

Figure 3\&4 Scenes in Hi Mom!

Fortunately, some women in the young generation are recognizing the important of the real self. Take the film Hi, Mom (2021) [5] by a female director Ling Jia as an instance. In addition to the outstanding plot and performance, Ling Jia presents a groundbreaking work that freed from the traditional male gaze. As one of the few young female directors in China, she depicts the scenes from the perspective of a female, which is rarely seen in popular Chinese films. After the film was released, many comments on the social media, most of which come from female audience, suggest that all the depictions in the film were both charming and comfortable. Although there are a plenty of close-up shots that directly focus on the female character's appearance, they do not provoke offense at all. The young mother does not put on appealing make-up or wear off-the-shoulder dress; the daughter does not have a slim and slender body, nor does she try to fit in a dress of the smallest size. Meanwhile, the movie does not describe a passionate love story between men and women like most popular movies; instead, it displays the relationship between two female figures (a mother and daughter). Since both of them are women, the demonstration of female beauty lies not in sexual attraction, but in the individual charm of a woman in the eyes of another woman. The purpose of this film is not to create exhibits that satisfy male aesthetics, but to reveal vivid female individuals.

\subsubsection{Conflicts Difficult to Resolve}

The emergence of this kind of work marks that young women in China are realising the oppressive nature of obedience to the male gaze and trying to reexamine and re-explore new subjectivities that are freed from the dominance of male expectations, desires, and constructs of femininity. Nonetheless, male gaze is not a thing that can be changed overnight by a single film. A large number of young Chinese women are not aware of this double consciousness and are constantly exposed to and impacted by male gaze. Indeed, many campaigns calling for gender equality are successful abroad, such as the "Me Too" movement. However, due to the profound influence brought by a highly patriarchal and feudal society lasting thousands of years in China' $s$ history, it is difficult for female generations to spontaneously realize the impact of this double consciousness on them. Various actions are needed to stimulate women' $\mathrm{s}$ self-awareness, self-esteem and self-recognition. The main goal at present should be to raise awareness of this long-rooted concept hidden in women' s subconscious, and then to avoid evaluating themselves from a male' $\mathrm{s}$ perspective. This would be a significant progress in Chinese society, for being able to define herself is the first step to protect the basic rights and dignity of a woman.

\section{CONCLUSION}

Male gaze is creating a kind of gender-related double consciousness upon young women in Chinese society, whose impact lies in various aspects, including body anxiety, workplace inequality, and conventional family roles. 
Once females adopt the perspective of males, not only would they try to fit themselves into this standard, but also would their confidence be undermined when they don' t meet such burdensome standards. Immersed in such an environment, women invisibly lose their own consciousness on themselves as well as their uniqueness of women as individuals.

However, despite some efforts to avoid double consciousness, its influence is still extensive and profound due to the long-lasting oppression towards women in past Chinese history. More needs to be done to strengthen women's sense of their own identity. The first thing to do is to raise the awareness of their subconscious double consciousness, and to avoid internalizing the influences of the male gaze.

\section{REFERENCES}

[1] L. Mulvey, Visual pleasure and narrative cinema. InVisual and other pleasures, Palgrave Macmillan, London, 1989, pp. 14-26. DOI: https://doi.org/10.1007/978-1-349-19798-9_3

[2] Tekanji., FAQ: What Is the "Male Gaze"? Finally, A Feminism 101 Blog, 26 Aug. 2007. Link: https://finallyfeminism101.wordpress.com/2007/08 /26/faq-what-is-the-\%E2\%80\%9Cmalegaze $\% \mathrm{E} 2 \% 80 \% 9 \mathrm{D} /$

[3] J. Berger, Ways of seeing BBC and Penguin Books. Harmondsworth, Middlesex, UK. 1972.

[4] Zhilian Recruitment, Investigation Report on the Status Quo of Chinese Women's Workplace in 2020. Link:

http://m.025ct.com/Home/Index/article/id/599095. html.\%20Accessed\%20April\%2010th,\%202021.

[5] J. Ling, Hi, Mom! Beijing JingxiCulture \&Tourism Co., Ltd., 2021. 